Article

\title{
The Influence of Essential Oil Compounds on Antibacterial Activity of Mupirocin-Susceptible and Induced Low-Level Mupirocin-Resistant MRSA Strains
}

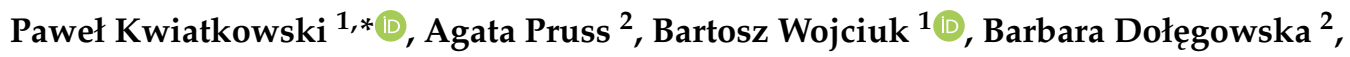 \\ Anna Wajs-Bonikowska ${ }^{3}$, Monika Sienkiewicz ${ }^{4}$, Monika Mężyńska ${ }^{5}$ and \\ Łukasz Łopusiewicz ${ }^{5}$ (D)
}

1 Department of Diagnostic Immunology; Chair of Microbiology, Immunology and Laboratory Medicine; Pomeranian Medical University in Szczecin; 72 Powstancow Wielkopolskich Avenue, 70-111 Szczecin, Poland

2 Department of Laboratory Medicine; Chair of Microbiology, Immunology and Laboratory Medicine; Pomeranian Medical University in Szczecin; 72 Powstancow Wielkopolskich Avenue, 70-111 Szczecin, Poland

3 Institute of General Food Chemistry, Faculty of Biotechnology and Food Sciences; Lodz University of Technology; Stefanowskiego 4/10, 90-924 Lodz, Poland

4 Department of Allergology and Respiratory Rehabilitation; Medical University of Lodz; Zeligowskiego 7/9, 90-752 Lodz, Poland

5 Center of Bioimmobilisation and Innovative Packaging Materials; Faculty of Food Sciences and Fisheries; West Pomeranian University of Technology Szczecin; Janickiego 35, 71-270 Szczecin, Poland

* Correspondence: pawel.kwiatkowski@pum.edu.pl; Tel.: +48-91-466-1659; Fax: +48-91-466-1652

Received: 25 July 2019; Accepted: 24 August 2019; Published: 27 August 2019

check for updates

\begin{abstract}
Because of the bacterial drug resistance development, it is reasonable to investigate chemical compounds capable of preventing the spread of resistance to mupirocin (MUP), commonly used in staphylococcal eradication. The objective of the study was to verify the influence of essential oil compounds (EOCs) on the antibacterial activity of MUP against mupirocin-susceptible (MupS) and induced low-level mupirocin-resistant (MupRL) methicillin-resistant Staphylococcus aureus (MRSA) strains. The following parameters were examined: MRSA ${ }^{\text {MupS }}$ and MRSA ${ }^{\text {MupRL }}$ susceptibility to EOCs (1,8-cineole, eugenol, carvacrol, linalool, (-)-menthone, linalyl acetate, and trans-anethole), the bacterial cell size distribution, and chemical composition by the use of Fourier Transform Infrared Spectroscopy (FTIR) and Raman spectroscopies. The MRSA ${ }^{\text {MupS }}$ and MRSA ${ }^{\text {MupRL }}$ strains were susceptible to all tested EOCs. 1,8-cineole and (-)-menthone showed synergistic activity against

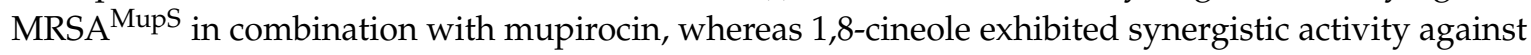
MRSA $^{\text {MupRL }}$ as well. In-depth analysis showed that both MRSA ${ }^{\text {MupS }}$ and MRSA ${ }^{\text {MupRL }}$ displayed similar distributions of the bacterial cell size. The FTIR and Raman spectra of the MRSA MupS and MRSA $^{\text {MupRL }}$ strains showed differences in some regions. New bands in the MRSA ${ }^{\text {MupRL }}$ Raman spectrum were observed. It was concluded that the use of 1,8-cineole in combination with mupirocin can increase the mupirocin activity against the MRSA ${ }^{\text {MupS }}$ and MRSA ${ }^{\text {MupRL }}$ strains.
\end{abstract}

Keywords: MRSA; mupirocin-susceptible; low-level mupirocin-resistant; essential oil compounds; synergistic activity; FTIR; Raman spectroscopy 


\section{Introduction}

Staphylococcus aureus carriage is common in human populations. Different body areas may be colonized, but these bacteria are usually found in the nose vestibule. Approximately $20 \%-30 \%$ of healthy adults are persistent carriers, about $50 \%$ are transient carriers, and $20 \%$ have never been colonized by S. aureus [1]. Colonization with S. aureus is a major risk factor for staphylococcal infections. It is estimated that approximately $80 \%$ of staphylococcal sepsis is of an endogenous origin [2]. Nasal carriage, hair follicle infection (furuncle), or ongoing bone inflammation may appear as the source of sepsis. Staphylococcal infections may also result from direct or indirect contact with a carrier (exogenous infection).

In order to eliminate the S. aureus carriage, as well as reduce the number of endogenous infections, topical mupirocin (an antibiotic produced by Pseudomonas fluorescens) ointment is applied to the nasal mucosa [3]. Decolonization is effective for patients who have completed the protocol, the duration of which typically ranges between 5 and 14 days [4]. The mechanism of mupirocin antibacterial activity is the inhibition of isoleucyl-tRNA synthetase (encoding by the ileS gene located on the chromosome) and associated bacterial protein synthesis blocking. There are two types of mupirocin resistance: low-level (MupRL, with a Minimum Inhibitory Concentration (MIC) between 8 and $256 \mathrm{mg} / \mathrm{L}$ ) and high-level (MupRH, with a MIC of $\geq 512 \mathrm{mg} / \mathrm{L}$ ). The MIC of mupirocin against susceptible strains is $\leq 4 \mathrm{mg} / \mathrm{L}$ [5]. MupRL is associated with ileS gene mutation. In turn, MupRH in S. aureus is mediated by a plasmid-encoded ileS2 gene, which encodes an alternate isoleucyl-tRNA synthetase, with a very low affinity towards mupirocin [6].

The effective eradication of $S$. aureus from the nasal vestibule reduces the amount of bacteria colonizing the skin surface. This prevents staphylococcal post-operative wound infections and, at the same time, reduces the amount of bacteria transferred to the hands of medical personnel $[7,8]$. At present, screening for $S$. aureus, in particular, methicillin-resistant S. aureus (MRSA), carriage and the eradication of these strains in the host is ordered in patients before cardiac surgery and orthopedic procedures. In addition, it is strongly recommended to perform a full-body bath with the addition of disinfectant (e.g., chlorhexidine, triclosan, or povidone-iodine) before relevant surgical procedures. This is in order to reduce the number of infections caused by this particular organism.

In the era of increasing resistance to antibiotics, it becomes a new challenge to the scientific community to search for compounds that reduce the virulence of $S$. aureus strains and possibly make their eradication easier. Essential oil compounds (EOCs) appear to be good candidates for field testing to determine the effectiveness of antibacterial activity against many bacterial species, both susceptible and resistant to antibiotics. Due to their characteristic taste and aroma, EOCs are widely used in perfume and flavoring industries. In addition, EOCs are also being applied in cosmetic as well as medicine industries [9]. The existing studies have shown that EOCs have got many antimicrobial, antioxidant, anti-inflammatory, cancer chemoprotective, allelopathic, repellent, and insecticidal activities [10].

The aim of the study was to investigate the influence of EOCs on the antibacterial activity of mupirocin against mupirocin-susceptible and induced low-level mupirocin-resistant MRSA strains.

\section{Results}

\subsection{The Antimicrobial Activity of Mupirocin against S. aureus ATCC 43300}

It was found that mupirocin showed antibacterial activity against the reference strain. The obtained MIC $\left(\mathrm{MIC}_{100}\right)$ value was $0.24 \pm 0.00 \mathrm{mg} / \mathrm{L}$. The $\mathrm{MIC}_{50}$ of mupirocin was calculated proportionally to the $\mathrm{MIC}_{100}$ value.

\subsection{Emergence of Mutations under MIC 50 of Mupirocin Pressure}

After seven days of exposure to the $\mathrm{MIC}_{50}(0.12 \mathrm{mg} / \mathrm{L})$ of mupirocin in Mueller-Hinton broth (MHB), it was noted that the reference strain showed an increase in its MIC, but remained in the susceptible range $(3.91 \mathrm{mg} / \mathrm{L})$. It was also found that the control strain in MHB without mupirocin had 
the same MIC value as described previously $(0.24 \mathrm{mg} / \mathrm{L})$. After this period, the new $\mathrm{MIC}_{50}(1.95 \mathrm{mg} / \mathrm{L})$ of mupirocin was calculated proportionally to the old MIC value as described above. This strain was exposed to the new $\mathrm{MIC}_{50}$ of mupirocin for seven additional days. It was again observed that exposure to the new $\mathrm{MIC}_{50}$ of mupirocin increased in the MIC during the second exposure and remained in the low-level resistance range $(31.25 \mathrm{mg} / \mathrm{L})$. After this time, the MIC of the control strain was still $0.24 \mathrm{mg} / \mathrm{L}$.

The strain susceptible to mupirocin was designated as $\mathrm{MRSA}^{\mathrm{MupS}}(\mathrm{MIC}=0.24 \mathrm{mg} / \mathrm{L}$ ), and with induced low-level mupirocin-resistance as $\mathrm{MRSA}^{\text {MupRL }}(\mathrm{MIC}=31.25 \mathrm{mg} / \mathrm{L})$.

\subsection{The Antimicrobial Effects of EOCs against MRSA ${ }^{M u p S}$ and MRSA $M u p R L$}

It was proven that both MRSA ${ }^{\text {MupS }}$ and MRSA MupRL strains were susceptible to all tested EOCs. The highest inhibiting activity was observed for carvacrol ( $\mathrm{MIC}=0.48 \pm 0.00-0.95 \pm 0.00 \mathrm{mg} / \mathrm{mL}$ ) In contrast, the lowest antibacterial activity of trans-anethole $(\mathrm{MIC}=494.0 \pm 0.0 \mathrm{mg} / \mathrm{mL})$ was revealed. The results of the MICs of EOCs against the MRSA ${ }^{\text {MupS }}$ and MRSA ${ }^{\text {MupRL }}$ strains are summarized in Table 1.

\subsection{Combination of EOCs and Mupirocin against MRSA $A^{M u p S}$ and $M R S A^{M u p R L}$}

The study indicated that 1,8-cineole showed synergistic activity in combination with mupirocin against both MRSA ${ }^{\text {MupS }}$ and MRSA ${ }^{\text {MupRL }}$ strains. Moreover, synergistic action was also demonstrated by (-)-menthone against the MRSA ${ }^{\text {MupS }}$ strain, in contrast to the MRSA MupRL (antagonistic effect). The following EOCs (eugenol, carvacrol, and trans-anethole) exhibited the additive effect in combination with mupirocin against the MRSA ${ }^{\text {MupRL }}$ strain, whereas the same EOCs (eugenol, carvacrol) presented antagonistic and indifferent effects against the MRSA ${ }^{\text {MupS }}$ strain. The results for the checkerboard assay against the MRSA ${ }^{\text {MupS }}$ and MRSA ${ }^{\text {MupRL }}$ strains are listed in Table 1.

\subsection{A Comparison of Cell Size between $M R S A^{M u p S}$ and $M R S A^{M u p R L}$}

It was found that the size of the bacterial colonies differed between the analyzed strains (susceptible to mupirocin and with induced low-level mupirocin-resistance) cultivated on Columbia agar with $5 \%$ sheep blood (Figure 1A). For this reason, it was assumed that the difference in colony size between these strains could be connected with the size of cells. However, an in-depth analysis showed that both MRSA $^{\text {MupS }}$ and MRSA ${ }^{\text {MupRL }}$ presented a similar distribution of the bacterial cell size, respectively $0.838 \pm 0.116 \mu \mathrm{m}$ for the susceptible and $0.806 \pm 0.132 \mu \mathrm{m}$ for the intermediate to mupirocin strain (Figure 1B). It was also observed using Scanning Electron Microscope (SEM) (Figure 1C).

\subsection{FTIR Analysis}

The FTIR spectra of the MRSA ${ }^{\mathrm{MupS}}$ and MRSA ${ }^{\text {MupRL }}$ strains are shown in Figure 2. The differences were observed particularly in the complex spectral region at $1700-1200 \mathrm{~cm}^{-1}$ and at $1200-900 \mathrm{~cm}^{-1}$. At $3279.03 \mathrm{~cm}^{-1}$ the absorbance value of MRSA ${ }^{\text {MupS }}$ was higher than MRSA ${ }^{\text {MupRL }}$. A noticeable growth of absorbance at bands $2960.96 \mathrm{~cm}^{-1}$ and $2925.90 \mathrm{~cm}^{-1}$ was observed. No changes were observed at $1634.58 \mathrm{~cm}^{-1}$. In the amide II region, MRSA MupRL absorbance increased at $1454.77 \mathrm{~cm}^{-1}$ and $1380.43 \mathrm{~cm}^{-1}$. At $1228.59 \mathrm{~cm}^{-1}$ and at $1058.71 \mathrm{~cm}^{-1}$, there was a decrease in absorbance in the MRSA $^{\text {MupS }}$ sample. It was also observed that absorbance increased in MRSA ${ }^{\text {MupRL }}$ in the narrow region centered at $935.13 \mathrm{~cm}^{-1}$.

\subsection{Raman Analysis}

The Raman spectra of the MRSA ${ }^{\text {MupS }}$ and MRSA MupRL strains are presented in Figure 3. Between 600 and $900 \mathrm{~cm}^{-1}$, centered around $775.0 \mathrm{~cm}^{-1}$, a higher band in the MRSA MupS sample was noted. A new band at $1155.0 \mathrm{~cm}^{-1}$ in the MRSA MupRL sample was observed. Higher bands at $1235.0 \mathrm{~cm}^{-1}$ and $1450.0 \mathrm{~cm}^{-1}$ were noticed in the MRSA MupRL sample in comparison to the MRSA MupS sample. In the region between 1500 and $1800 \mathrm{~cm}^{-1}$, a new band at $1525.0 \mathrm{~cm}^{-1}$ was noted in the MRSA MupRL 
sample, whereas in both samples, a similar intensity of band at $1655.0 \mathrm{~cm}^{-1}$ was observed. In the region between 2800 and $3100 \mathrm{~cm}^{-1}$, an intense signal was noted for the MRSA MupRL sample.

Table 1. Fractional inhibitory concentration (FIC) and FIC indices (FICI) of mupirocin-essential oil compound (EOC) pairs against mupirocin-susceptible $\left(\mathrm{MRSA}^{\mathrm{MupS}}\right)$ and induced low-level mupirocin-resistant (MRSA ${ }^{\text {MupRL }}$ ) strains.

\begin{tabular}{|c|c|c|c|c|c|c|}
\hline Strains & Mupirocin-EOCs & $\mathrm{MIC}_{\mathrm{O}}$ & $\mathrm{MIC}_{\mathrm{C}}$ & FIC & FICI & Type of Interaction \\
\hline \multirow{21}{*}{ MRSA $^{\text {MupS }}$} & \multicolumn{6}{|c|}{ Mupirocin-1,8-cineole } \\
\hline & Mupirocin (mg/L) & $0.24 \pm 0.00$ & $0.06 \pm 0.00$ & 0.25 & \multirow[b]{2}{*}{0.44} & \multirow{2}{*}{ synergy } \\
\hline & 1,8-cineole $(\mathrm{mg} / \mathrm{mL})$ & $307.00 \pm 132.93$ & $57.56 \pm 0.00$ & 0.19 & & \\
\hline & \multicolumn{6}{|c|}{ Mupirocin-eugenol } \\
\hline & Mupirocin (mg/L) & $0.24 \pm 0.00$ & $0.24 \pm 0.00$ & 1.0 & \multirow[b]{2}{*}{5.0} & \multirow{2}{*}{ antagonism } \\
\hline & Eugenol (mg/mL) & $2.08 \pm 0.00$ & $8.34 \pm 0.00$ & 4.0 & & \\
\hline & \multicolumn{6}{|c|}{ Mupirocin-carvacrol } \\
\hline & Mupirocin (mg/L) & $0.24 \pm 0.00$ & $0.24 \pm 0.00$ & 1.0 & \multirow[b]{2}{*}{5.0} & \multirow{2}{*}{ antagonism } \\
\hline & Carvacrol (mg/mL) & $0.95 \pm 0.00$ & $3.81 \pm 0.00$ & 4.0 & & \\
\hline & \multicolumn{6}{|c|}{ Mupirocin-linalool } \\
\hline & Mupirocin (mg/L) & $0.24 \pm 0.00$ & $0.24 \pm 0.00$ & 1.0 & \multirow[b]{2}{*}{1.5} & \multirow[b]{2}{*}{ indifference } \\
\hline & Linalool $(\mathrm{mg} / \mathrm{mL})$ & $6.8 \pm 1.0$ & $3.4 \pm 0.0$ & 0.5 & & \\
\hline & \multicolumn{6}{|c|}{ Mupirocin-(-)-menthone } \\
\hline & Mupirocin (mg/L) & $0.24 \pm 0.00$ & $0.06 \pm 0.00$ & 0.25 & \multirow[b]{2}{*}{0.38} & \multirow{2}{*}{ synergy } \\
\hline & (-)-Menthone (mg/mL) & $27.91 \pm 0.00$ & $3.49 \pm 0.00$ & 0.13 & & \\
\hline & \multicolumn{6}{|c|}{ Mupirocin-linalyl acetate } \\
\hline & Mupirocin (mg/L) & $0.24 \pm 0.00$ & $0.12 \pm 0.00$ & 0.5 & \multirow[b]{2}{*}{1.5} & \multirow[b]{2}{*}{ indifference } \\
\hline & Linalyl acetate $(\mathrm{mg} / \mathrm{mL})$ & $450.5 \pm 0.0$ & $450.5 \pm 0.0$ & 1.0 & & \\
\hline & \multicolumn{6}{|c|}{ Mupirocin-trans-anethole } \\
\hline & Mupirocin (mg/L) & $0.24 \pm 0.00$ & $0.24 \pm 0.00$ & 1.0 & \multirow{2}{*}{2.0} & \multirow{2}{*}{ indifference } \\
\hline & trans-Anethole $(\mathrm{mg} / \mathrm{mL})$ & $494.0 \pm 0.0$ & $494.0 \pm 0.0$ & 1.0 & & \\
\hline & \multicolumn{6}{|c|}{ Mupirocin-1,8-cineole } \\
\hline & Mupirocin (mg/L) & $31.25 \pm 0.00$ & $0.98 \pm 0.00$ & 0.03 & \multirow{2}{*}{0.28} & syneroy \\
\hline & 1,8-cineole $(\mathrm{mg} / \mathrm{mL})$ & $57.56 \pm 0.00$ & $14.39 \pm 0.00$ & 0.25 & & synergy \\
\hline & & & irocin-eugeno & & & \\
\hline & Mupirocin (mg/L) & $31.25 \pm 0.00$ & $15.63 \pm 0.00$ & 0.5 & & \\
\hline & Eugenol (mg/mL) & $8.34 \pm 0.00$ & $4.17 \pm 0.00$ & 0.5 & 1.0 & addition \\
\hline & & $\mathrm{Mu}$ & rocin-carvacr & & & \\
\hline & Mupirocin (mg/L) & $31.25 \pm 0.00$ & $15.63 \pm 0.00$ & 0.50 & & \\
\hline & Carvacrol $(\mathrm{mg} / \mathrm{mL})$ & $0.48 \pm 0.00$ & $0.12 \pm 0.00$ & 0.25 & 0.75 & addition \\
\hline & & & irocin-linaloo & & & \\
\hline MRSA $^{\text {MupRL }}$ & Mupirocin (mg/L) & $31.25 \pm 0.00$ & $62.5 \pm 0.0$ & 2.0 & & \\
\hline & Linalool (mg/mL) & $2.83 \pm 0.98$ & $27.19 \pm 0.00$ & 9.6 & 11.6 & antagonism \\
\hline & & Mup & cin-(-)-menth & & & \\
\hline & Mupirocin (mg/L) & $31.25 \pm 0.00$ & $62.5 \pm 0.0$ & 2.0 & & antaoonism \\
\hline & (-)-Menthone (mg/mL) & $6.98 \pm 0.00$ & $111.63 \pm 0.00$ & 16.0 & 18.0 & antagonism \\
\hline & & Mupi & cin-linalyl ace & & & \\
\hline & Mupirocin (mg/L) & $31.25 \pm 0.00$ & $31.25 \pm 0.00$ & 1.0 & & \\
\hline & Linalyl acetate $(\mathrm{mg} / \mathrm{mL})$ & $450.5 \pm 0.0$ & $450.5 \pm 0.0$ & 1.0 & 2.0 & indifference \\
\hline & & Mupi & cin-trans-anet & & & \\
\hline & Mupirocin (mg/L) & $31.25 \pm 0.00$ & $7.81 \pm 0.00$ & 0.25 & & \\
\hline & trans-Anethole $(\mathrm{mg} / \mathrm{mL})$ & $494.0 \pm 0.0$ & $247.0 \pm 0.0$ & 0.49 & 0.74 & addition \\
\hline
\end{tabular}

Values are expressed as mean \pm standard deviation. MICo, MIC of EOC or mupirocin; MICc, MIC of EOC/mupirocin combination. FIC index $=$ FIC of EOC + FIC of mupirocin. FICI $<0.5$, synergy; $0.5 \leq$ FICI $\leq 1.0$, addition; $1.1<$ FICI $\leq 4.0$, indifference; FICI $>4.0$, antagonism. Using the known concentrations of EOCs, the final result was expressed in $\mathrm{mg} / \mathrm{mL}$. 
a)

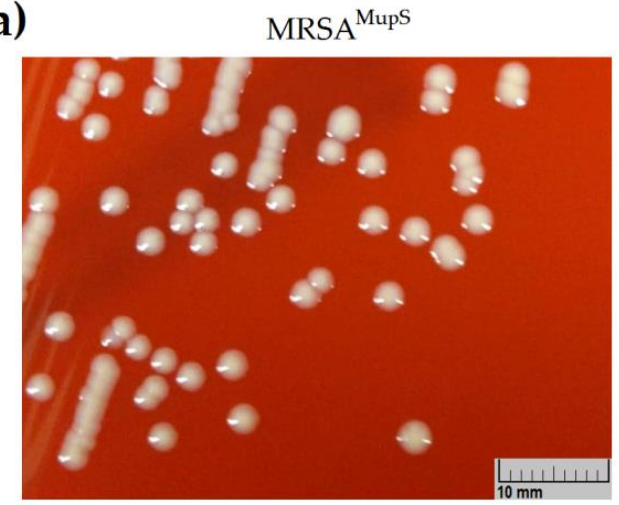

MRSA MupRL

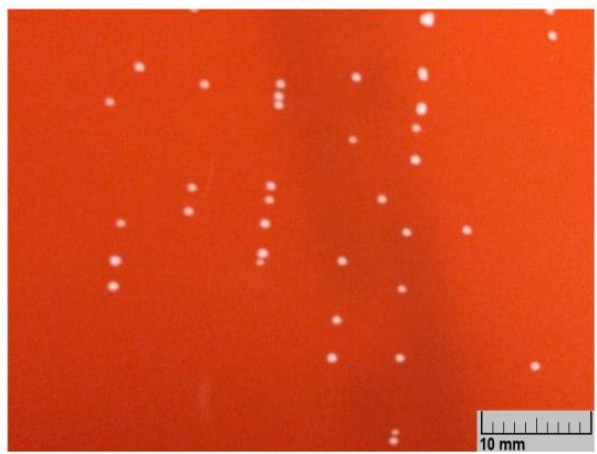

b)

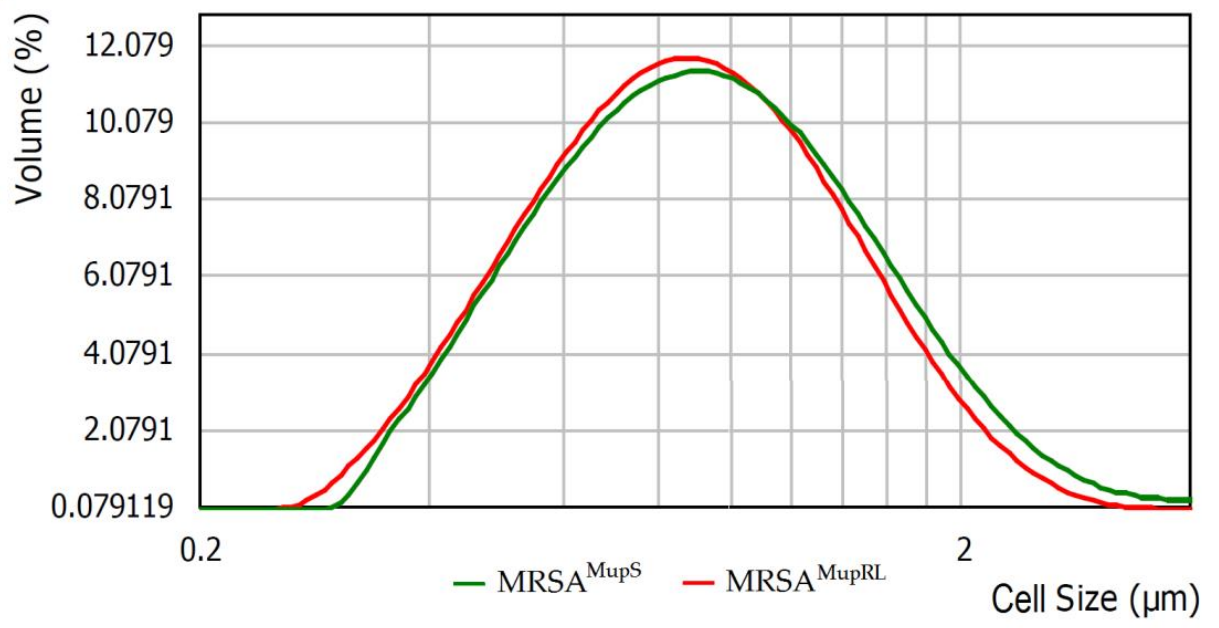

c)
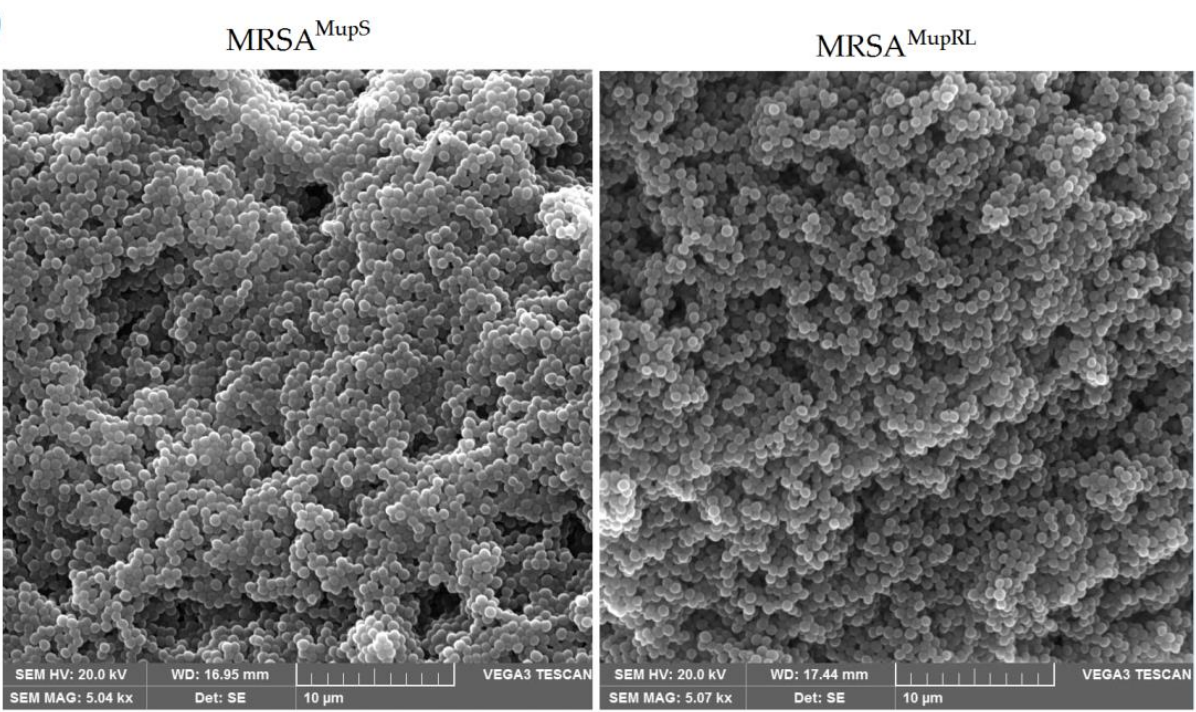

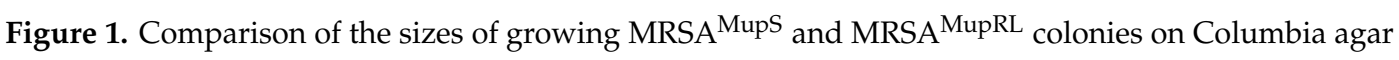
with 5\% sheep blood (a), and cells using Mastersizer 2000 (b), and SEM (c). 


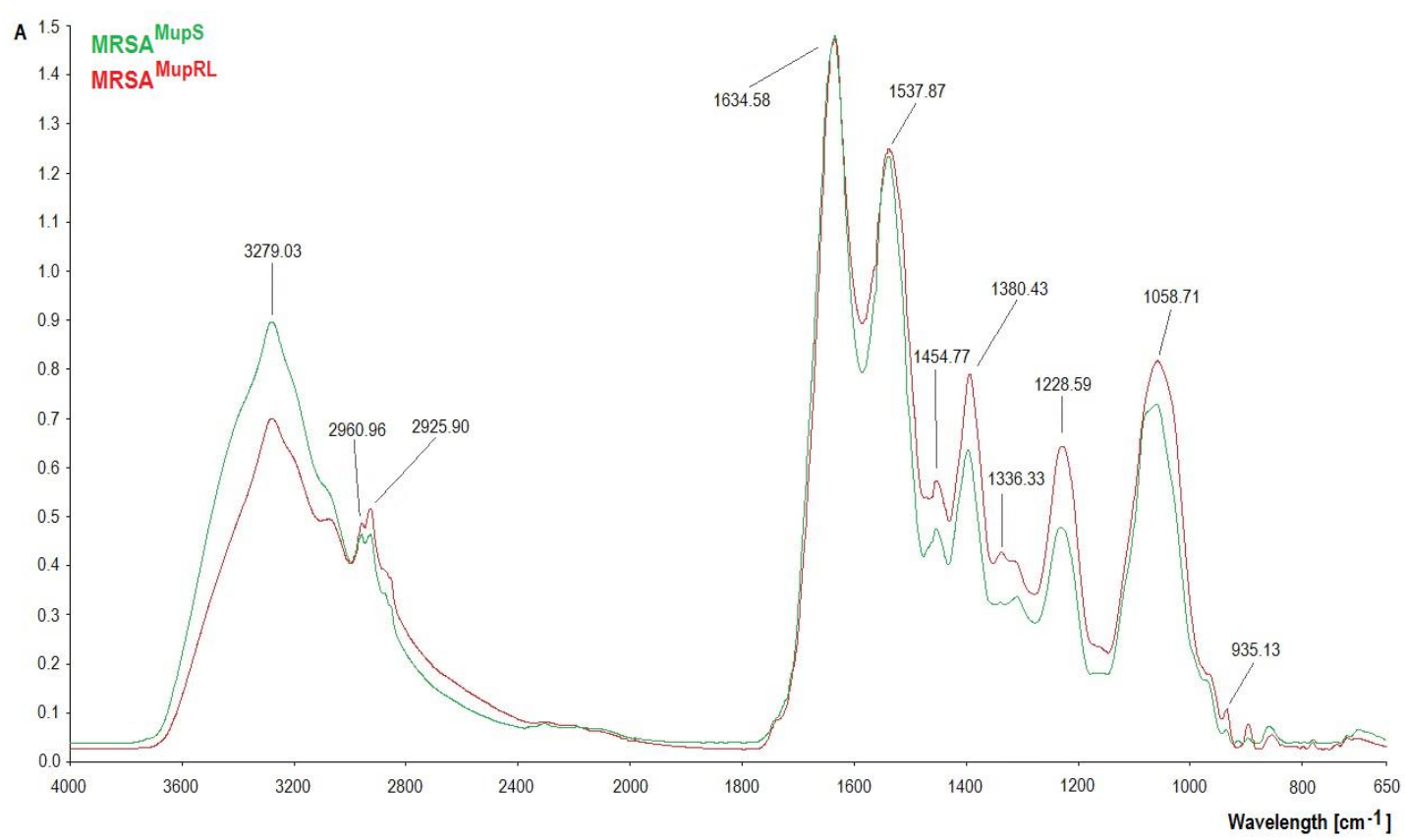

Figure 2. FTIR spectra of MRSA MupS and MRSA MupRL strains.

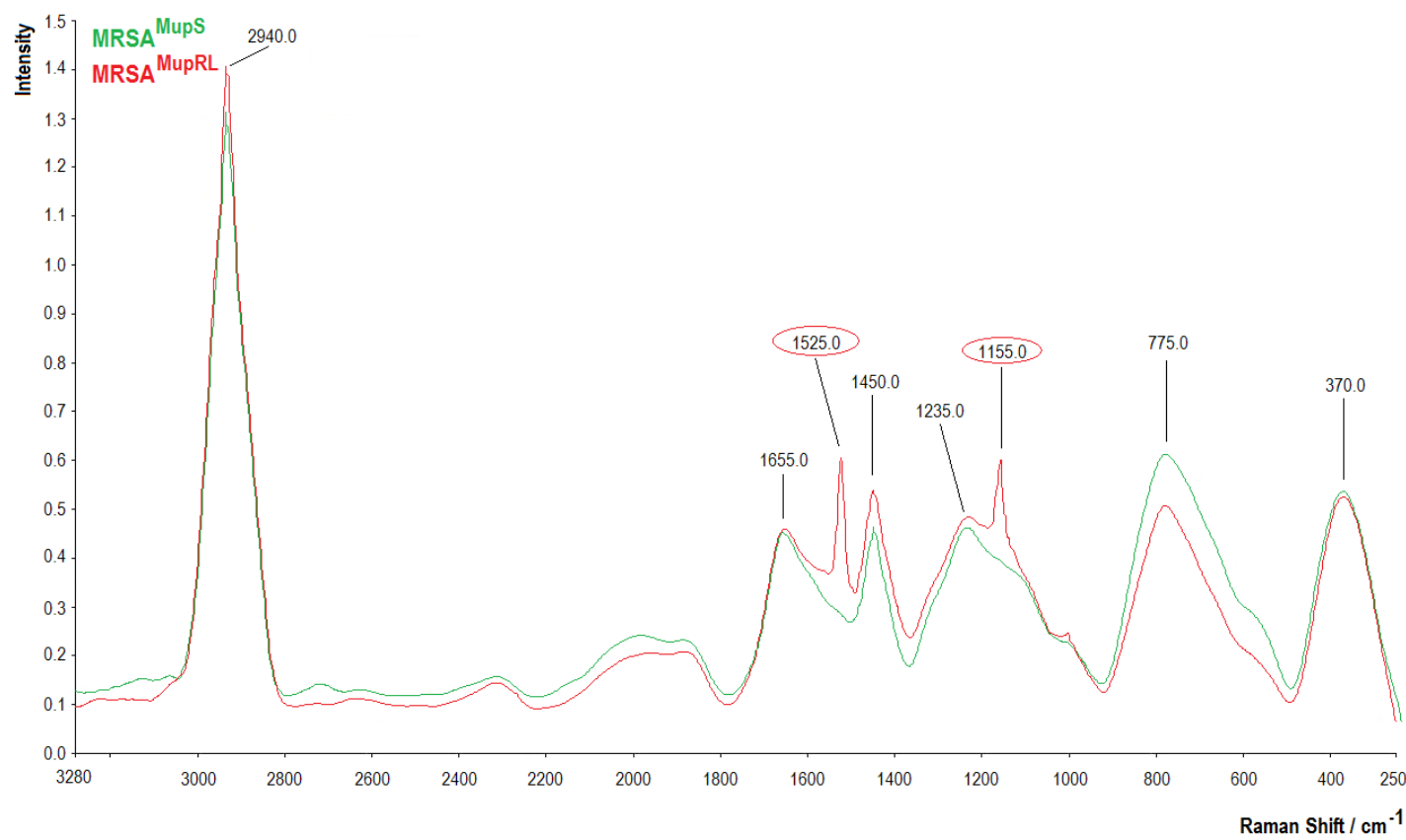

Figure 3. Raman spectra of MRSA $\mathrm{MupS}^{\mathrm{M}}$ and MRSA $\mathrm{MupRL}^{\mathrm{M}}$ strains.

\section{Discussion}

Scientific research with modern methodology has confirmed the valuable properties of many medicinal plants known for ages in medicine. The development of accompanying sciences has led to research on plant-originated compounds, providing reliable scientific data. There has been a lot of research that has proven multidirectional activity and the potential of plant-derived compounds. Essential oils-secondary metabolites of plants-have been of increasing interest in recent years. According to Nazzaro et al., essential oils are classified as "Generally Recognised as Safe" (GRAS) by the Food and Drugs Administration (FDA). These are not harmful and are more widely accepted by consumers [11]. These represent anticancer and anti-diabetic activities and are recommended 
for cardiovascular diseases including atherosclerosis and thrombosis [12]. In this research, the main constituents of the following essential oils were studied: Eucalyptus globulus Labill. (1,8-cineole, content not less than 70\%), Syzygium aromaticum (L.) Merr. \& Perry, Eugenia caryophyllata Thunb. (eugenol, required from $75 \%$ to $88 \%$ ), Lavandula angustifolia Mill. (required: linalool from $20 \%$ to $45 \%$ and linalyl acetate from $25 \%$ to $46 \%$ ), Mentha $\times$ piperita L. (menthone required from $14 \%$ to $32 \%$ ), Foeniculum vulgare Mill. (trans-anethole required from $60 \%$ to $80 \%$ ), and Thymus vulgaris L. (carvacrol, required 40\%) [13]. Because of their high antimicrobial activity, they bring hope to stop the growth of resistance among pathogenic microorganisms to synthetic drugs. Combinatorial studies, which involve essential oil blends, showed their synergistic action against multidrug-resistant pathogens like MRSA, Escherichia coli, producing extended spectrum beta-lactamases, and also against yeasts or dermatophytes [14-16].

The prevalence of high and low-level mupirocin resistance affects not only $S$. aureus, but also coagulase-negative staphylococci-a reservoir of conjugative plasmids of mupirocin resistance frequently responsible for serious infections, especially in immunocompromised hosts and patients treated with implants and catheters [17]. Due to the easy spread of staphylococci in the hospital environment and their ability to survive on non-living surfaces, it is reasonable to look for compounds that will prevent the spread of resistance to mupirocin used for staphylococcal eradication in patients and hospital staff. Data collected by Khoshnood et al. showed that the emergence of mupirocin resistance is currently increasing mostly among MRSA isolates [18].

In this study the influence of the following essential oil dominant constituents (1,8-cineole, eugenol, carvacrol, linalool, (-)-menthone, linalyl acetate, and trans-anethole) on the antibacterial activity of mupirocin against mupirocin-susceptible and induced low-level mupirocin-resistant MRSA strains was investigated. This investigation was further deepened by the spectral analysis of the tested strains believing that changes in the structures of outer bacterial cell membranes may affect the penetration of essential oil components and, in consequence, the mupirocin activity.

This research showed the differences in FTIR and Raman spectra between MRSA MupS and MRSA $^{\text {MupRL }}$ strains. The differences in FTIR spectra between MRSA MupS and MRSA ${ }^{\text {MupRL }}$ strains were observed particularly in the complex spectral region at $1700-1200 \mathrm{~cm}^{-1}$ where many types of vibrations were present $\left(\mathrm{CH}_{2}\right.$ bending, $\mathrm{COO}$ - stretching, $\mathrm{PO}_{2}^{-}$stretching) and at $1200-900 \mathrm{~cm}^{-1}$, corresponding to the stretching and bending vibrations of $\mathrm{C}-\mathrm{O}-\mathrm{C}$ and $\mathrm{C}-\mathrm{OH}$ groups and the $\mathrm{C}-\mathrm{O}$ ring vibrations of the polysaccharide region attributed to carbohydrates in the bacterial cell wall [19]. At $3279.03 \mathrm{~cm}^{-1}$, the absorbance value of MRSA MupS was higher than MRSA MupRL, suggesting more hydroxyl groups in the MRSA MupS strain. A noticeable growth of absorbance at bands $2960.96 \mathrm{~cm}^{-1}$ and $2925.90 \mathrm{~cm}^{-1}$, respectively, was attributed to $\mathrm{CH}_{2}$ and $\mathrm{CH}_{3}$ groups. No changes were observed at $1634.58 \mathrm{~cm}^{-1}$ (amide I), attributed to proteins. In the amide II region, MRSA MupRL absorbance increased at $1454.77 \mathrm{~cm}^{-1}$ and $1380.43 \mathrm{~cm}^{-1}$, the bands that are attributed to the asymmetric bending of methyl groups in proteins and lipids, and stretching the C-N vibrations of cytosine-guanine pairs. At $1228.59 \mathrm{~cm}^{-1}$, imputed to the asymmetric stretching of the phosphodiester backbone, there was a decrease in absorbance in MRSA MupS [20]. At $1058.71 \mathrm{~cm}^{-1}$, which was attributed to stretching $\mathrm{OH}$ coupled with bending the $\mathrm{CO}$ of the polysaccharides capsule and peptidoglycan and stretching the $\mathrm{CO}$ of polysaccharides, a decline in MRSA ${ }^{\text {MupS }}$ absorbance was noted [21]. The absorbance increase in

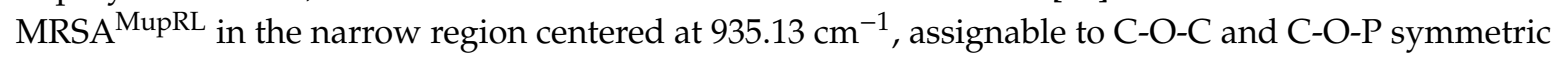
stretching in cell wall oligosaccharides and polysaccharides, was also noticed [20].

The differences in the Raman spectra between the MRSA ${ }^{\text {MupS }}$ and MRSA MupRL strains were observed between 600 and $900 \mathrm{~cm}^{-1}$, centered around $775 \mathrm{~cm}^{-1}$, a noticeably higher band in the

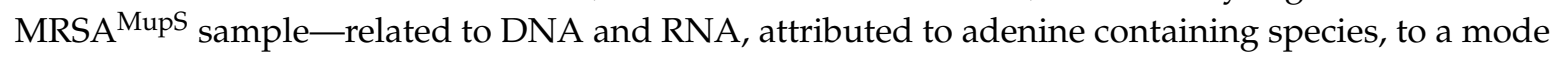
of adenine ring breathing in DNA and DNA phosphodiester stretching, out-of-plane ring breathing modes of tyrosine, and to the C-O-C stretching vibration of 1,4 glycosidic link in carbohydrates [22,23]. The region between 900 and $1500 \mathrm{~cm}^{-1}$ was characterized by a series of emission bands from amides, lipids, and carbohydrates. A new band at $1155 \mathrm{~cm}^{-1}$ in MRSA MupRL was related to C-O-C and $=\mathrm{C}-\mathrm{C}=$ antisymmetric stretching in aliphatic esters and the glycosidic link of carbohydrates. The band 
$1235 \mathrm{~cm}^{-1}$ higher in the MRSA MupRL sample was related to C-N stretching in the amide III region [23]. At $1450 \mathrm{~cm}^{-1}$, the higher intensity in the MRSAMupRL sample can be attributed to $\mathrm{CH}_{2}$ vibrations from membrane lipids and polysaccharides such as poly-N-acetylglucosamine. The region between 1500 and $1800 \mathrm{~cm}^{-1}$ contained one $\alpha$-helix-related band $(C=O$ and $-C=C$ stretch $)$ at $1525 \mathrm{~cm}^{-1}$ in the MRSA ${ }^{\text {MupRL }}$ sample. In both samples, a similar intensity of the $\beta$-sheet-related $C=\mathrm{O}$ stretching emission of amide I (proteins) and in lipids at $1655 \mathrm{~cm}^{-1}$ was noted $[23,24]$. In the region between 2800 and $3100 \mathrm{~cm}^{-1}$, a more intense signal was noticed for the MRSAMupRL sample, attributed to the symmetric and antisymmetric stretching of $\mathrm{CH}_{2}$ and $\mathrm{CH}_{3}$ in lipids, fatty acids, proteins, and carbohydrates [23].

The mupirocin molecule consists of 9-hydroxy nonanoic acid (a short fatty acid side-chain) and monic acid (a C polyketide-derived substructure), which are linked by an unsaturated ester linkage [18]. Presenting a negative charge, mupirocin enters the bacterial cells by passive diffusion in an energy-independent manner $[25,26]$. The pivotal factors which determine the resistance to mupirocin are potentially the following: lower affinity to the antibiotic, higher affinity to the substrate, overproduction of the synthetase enzyme, decreased permeability of the bacterial membranes, or a combination of these $[18,25]$. Based on the spectral analysis of the MRSA ${ }^{\text {MupS }}$ and MRSA ${ }^{\text {MupRL }}$ strains, it can be concluded that resistance to mupirocin may affect the chemical composition of bacterial cells. Those changes, particularly related to the polysaccharides and peptidoglycan of the capsule, as well as cell wall oligosaccharides and polysaccharides, may result in different electrostatic interactions with anionic antibiotic molecules, leading to its lower penetration into the MRSA ${ }^{\text {MupRL }}$ strain.

The current research confirmed the thesis of the various activities of essential oils and their components in combination with antibacterial compounds against susceptible and resistant bacteria of the same species. Our previous study showed that trans-anethole appeared efficient in increasing susceptibility to mupirocin and decreasing biofilm formation in mupirocin-resistant $S$. aureus strains [27]. It has also been observed in this study. Trans-Anethole presented indifferent and addictive effects

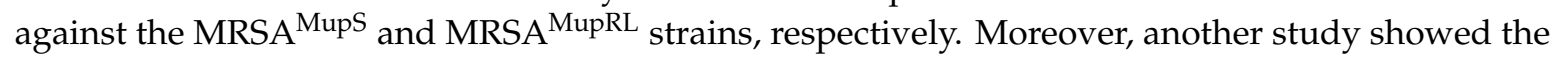
good antibacterial activity of terpenoids against the MRSA ${ }^{\text {MupS }}$ and MRSA ${ }^{\text {MupRL }}$ strains and also confirmed the special properties of 1,8-cineole as a recognized permeation enhancer, described by Hendry et al. in his research on MRSA [28]. Kifer et al. did not notice the difference in the activity of EOCs (menthol and 1,8-cineole) towards mupirocin in planktonic MRSA strains, but the mixture of mupirocin and thymol had an inconclusive effect. They also observed that 1,8-cineole exerted a potentiated biofilm-eliminating effect [29]. Other research also showed the increased antimicrobial activity of 1,8-cineole in combination with chlorhexidine gluconate (CHG) against MRSA strains [30]. According to $\mathrm{Xu}$ et al., E. globulus essential oil contains mainly 1,8-cineole. This is recommended as a good remediation against respiratory infectious diseases, also for children, and is a component of many medicinal mixtures. The authors observed that a dose range of $0-64.15 \mathrm{mg} / \mathrm{kg} /$ day did not cause even slight organ damage in mice [31]. The current study has shown that the most effective combination of 1,8-cineole and mupirocin decreased the MIC of 1,8-cineole from $307.00 \pm 132.93$ to $57.56 \pm 0.00 \mathrm{mg} / \mathrm{mL}$ and from $57.56 \pm 0.00$ to $14.39 \pm 0.00 \mathrm{mg} / \mathrm{mL}$, respectively, for the MRSA ${ }^{\text {MupS }}$ and MRSA ${ }^{\text {MupRL }}$ strains. Yadav et al. showed the very good antibacterial activity of eugenol, alone or in combination with carvacrol against methicillin-susceptible S. aureus (MSSA) and MRSA clinical strain biofilms. They found that eugenol significantly decreased the expression of biofilm- and enterotoxin-related genes, and decreased bacterial colonization in the middle ear as well. The authors recommended that eugenol be administered alone or in combination with carvacrol in S. aureus biofilm-related infections. Moreover, eugenol is approved as a safe food preservative by the European Union and FDA. It has been extensively applied in stomatology as well [32].

Despite the evident differences in subcellular structures between the two tested strains, no particular differences were observed regarding their physical properties apart from colony size. Moreover, these revealed similar susceptibility to the tested combinations of mupirocin with particular EOCs. Trombetta et al. proposed a mechanism of the antimicrobial effect of three monoterpenes 
(menthol, thymol, and linalyl acetate) [33]. These compounds may be responsible for the membrane permeability and leakage of intracellular materials. Furthermore, the authors supposed that these effects could be dependent on the lipid composition and net surface charge of microbial membranes. The study showed that the differences between FTIR and Raman spectra provided by both strains indicate cellular wall variability, which may possibly contribute both to mupirocin and EOCs' susceptibility. It is clearly visible in the mupirocin-(-)-menthone combination, which showed synergy and an antagonistic effect against MRSA ${ }^{\text {MupS }}$ and MRSA ${ }^{\text {MupRL }}$, respectively.

Still more precise fingerprinting methods are needed to identify the therapeutic targets for new drugs or new formulas of known drugs, e.g., combinations between the conventional antibiotics and EOCs. Targeting molecular patterns is appearing outstandingly in emerging studies on drugs like mupirocin, which is not decisive for survival in individual cases but represents a significant tool in preventive procedures in terms of the spread of drug resistance.

\section{Materials and Methods}

\subsection{Bacterial Strain and Growth Conditions}

The S. aureus ATCC 43300 (MRSA) was used in this study. The reference strain was cultivated for $18 \mathrm{~h}$ at $37^{\circ} \mathrm{C}$ on Columbia agar with $5 \%$ sheep blood (bioMérieux, Warsaw, Poland) and then a single colony was inoculated into Mueller-Hinton broth (MHB, Sigma-Aldrich, Darmstadt, Germany) and cultured for $18 \mathrm{~h}$ at $37^{\circ} \mathrm{C}$.

\subsection{Chemicals}

Concentrations of mupirocin ( $\geq 92 \%$ purity, obtained through HPLC, Sigma-Aldrich, Germany) from 250 to $0.12 \mathrm{mg} / \mathrm{L}$ were prepared by dissolving the drug in dimethyl sulfoxide (DMSO, Loba Chemie, Mumbai, India) $(2 \%, v / v)$ and diluting by MHB. Concentrations of EOCs-1,8-cineole (99\% purity, Ernesto Ventos S.A., Barcelona, Spain), eugenol ( $\geq 98 \%$ purity, Ernesto Ventos S.A., Spain), carvacrol (99\% purity, Sigma-Aldrich, Germany), linalool ( $\geq 96 \%$ purity, Ernesto Ventos S.A., Spain), (-)-menthone (96\% purity, Ernesto Ventos S.A., Spain), linalyl acetate ( $\geq 97 \%$ purity, Ernesto Ventos S.A., Spain), and trans-anethole (99\% purity, Sigma-Aldrich, Germany) from 500 to $0.12 \mu \mathrm{L} / \mathrm{mL}-$ were prepared by dissolving compounds in Tween 80 (Sigma-Aldrich, Germany) (1\%,v/v) and diluting by MHB.

\subsection{Determination of Minimum Inhibitory Concentration (MIC) of Mupirocin against S. aureus ATCC 43300}

The MIC of mupirocin was determined by the broth microdilution method in a 96-well microplate according to the Clinical and Laboratory Standards Institute [34]. Each well contained $50 \mu \mathrm{L}$ of the appropriate mupirocin concentration and $50 \mu \mathrm{L}$ of the S. aureus ATCC 43300 suspension at a final concentration of $10^{6} \mathrm{CFU} / \mathrm{mL}$. Staphylococcal suspension was performed for 18-hour cultures using MHB. The MIC was estimated as described earlier [35]. To exclude an inhibitory effect of $2 \%(v / v)$ DMSO on the reference strain growth, the control assays with MHB and MHB supplemented with DMSO were performed. All tests were carried out in triplicate.

\subsection{Induction of Mutations}

The induction of mutations in the S. aureus ATCC 43300 strain was performed according to Lee et al. [36] with minor modification. S. aureus ATCC 43300 was incubated in MHB (control) and MHB supplemented with the $\mathrm{MIC}_{50}$ of mupirocin according to the original MIC of the reference strain. After $18 \mathrm{~h}$ of incubation at $37^{\circ} \mathrm{C}$, the suspensions were adjusted to a $0.5 \mathrm{McFarland}$ scale and diluted again (1:100) in fresh MHB. This assay was repeated on a daily basis for seven days to reflect the duration of in vivo exposure to mupirocin in eradication protocols from the nose vestibule (about 14 days). Then the reference strain underwent MIC determinations by the broth microdilution method described above. After seven days, the strain was subjected to another seven-day mupirocin 
exposure cycle, with the drug concentration adjusted according to the new MIC. The obtained MIC was estimated by the broth microdilution method as described previously (in Section 4.3). At this stage, the strain susceptible to mupirocin (control) was designated as MRSA ${ }^{\text {MupS }}$, and with induced low-level mupirocin resistance as MRSA ${ }^{\text {MupRL }}$.

\subsection{Determination of MIC of EOCs against MRSA $A^{M u p S}$ and MRSA $A^{M u p R L}$ Strains}

The MIC values of the following EOCs (1,8-cineole, eugenol, carvacrol, linalool, (-)-menthone, linalyl acetate, and trans-anethole) against the MRSA ${ }^{\text {MupS }}$ and MRSA ${ }^{\text {MupRL }}$ strains were determined by the broth microdilution method according to Clinical and Laboratory Standards Institute (CLSI) [34] with a minor modification: a final concentration of sterile 1\% $(v / v)$ Tween 80 was incorporated into MHB to enhance the EOCs' solubility. All tests were performed as described above (in Section 4.3). To exclude an inhibitory effect of $1 \%(v / v)$ Tween 80 on the MRSA MupS and MRSA ${ }^{\text {MupRL }}$ strains' growth, the control assays with MHB and MHB supplemented with 1\% (v/v) Tween 80 were performed. Using the known concentrations of EOCs, the final result was expressed in $\mathrm{mg} / \mathrm{mL}$.

\subsection{Checkerboard Method}

The checkerboard method was performed against the MRSA ${ }^{\text {MupS }}$ and MRSA ${ }^{\text {MupRL }}$ strains as previously described [37]. Two-fold dilutions (250-0.12 mg/L for mupirocin and 500-0.12 $\mu \mathrm{L} / \mathrm{mL}$ for each EOC) were performed. Each well contained the following components: $25 \mu \mathrm{L}$ of the appropriate concentration of EOC, $25 \mu \mathrm{L}$ of the appropriate mupirocin concentration, and $50 \mu \mathrm{L}$ of bacterial suspension containing the final concentration of $10^{6} \mathrm{CFU} / \mathrm{mL}$ in each well. The plates were incubated at $37^{\circ} \mathrm{C}$ for $18 \mathrm{~h}$. All assessments were performed in duplicates. Using the known densities of the EOCs, the final result was expressed in $\mathrm{mg} / \mathrm{mL}$. The MIC of both mupirocin and the EOCs combined or without combination were determined using resazurin as described in the previous study [35]. The combined effects of mupirocin and the EOCs were calculated and expressed in terms of a fractional inhibitory concentration index (FICI) using the following formula:

$$
\begin{gathered}
\text { FIC }=\frac{\text { MIC of EOC or mupirocin in combination }}{\text { MIC of EOC or mupirocin alone }} \\
\text { FICI }=\text { FIC of EOC }+ \text { FIC of mupirocin }
\end{gathered}
$$

Results were interpreted as follows: synergy (FICI $<0.5)$, addition $(0.5 \leq \mathrm{FICI} \leq 1.0)$, indifference $(1.1<\mathrm{FICI} \leq 4.0)$, or antagonism $(\mathrm{FICI}>4.0)$ [37].

\subsection{Bacterial Cell Size Distribution}

The bacterial cell size distribution was analyzed by the use of the particle size analyzer Mastersizer 2000 (Malvern Panalytical Ltd., Malvern, United Kingdom). The bacteria (MRSA ${ }^{\text {MupS }}$ and MRSA ${ }^{\text {MupRL}}$ ) were cultivated for $18 \mathrm{~h}$ at $37^{\circ} \mathrm{C}$ on Columbia agar with $5 \%$ sheep blood. After incubation, grown colonies were harvested with a loop and suspended in sterile phosphate-buffered saline (PBS, pH 7.4). Cells were centrifuged (5000 rpm for $5 \mathrm{~min}$ ) and washed three times with PBS. After this, bacteria were suspended initially in PBS with $0.01 \%$ sodium dodecyl sulfate solution (SDS, Sigma-Aldrich, Germany) to reach concentration 7 in a McFarland scale (approx. $2.1 \times 10^{9} \mathrm{CFU} / \mathrm{mL}$ ), then vigorously vortexed for $1 \mathrm{~min}$. The bacterial suspensions were then dispersed in $800 \mathrm{~mL}$ (stirrer speed-2000 rpm) of distilled water at room temperature $\left(20^{\circ} \mathrm{C}\right)$ to reach obscurance in range $5.0 \%$. Each sample was measured in triplicate.

\subsection{A Determination of Functional Groups in Staphylococcal Cells by the Use of Fourier Transform Infrared (FTIR) and Raman Spectroscopy}

In order to confirm the presence of particular chemical moieties in MRSA MupS as well as MRSA $^{\text {MupRL }}$ cells, FTIR and Raman spectroscopy analyses were carried out. After the cultivation of 
these strains for $18 \mathrm{~h}$ at $37^{\circ} \mathrm{C}$ on Columbia agar with $5 \%$ sheep blood, the cells were washed three times by $5 \mathrm{~mL}$ of PBS, centrifuged at 5000 for $5 \mathrm{~min}$, and dried for $24 \mathrm{~h}$ at $37^{\circ} \mathrm{C}$. The FTIR spectra of bacterial cells dry samples were obtained at room temperature by attenuated total reflection with a FTIR spectrometer (Perkin Elmer Spectrophotometer 100, Waltham, MA, USA). The samples (100 mg) were then scanned at a range between $650 \mathrm{~cm}^{-1}$ and $4000 \mathrm{~cm}^{-1}$ (64 scans and $1 \mathrm{~cm}^{-1}$ resolution). The obtained spectra were normalized, baseline corrected, and analyzed using SPECTRUM software (v10, Perkin Elmer Spectrophotometer, Waltham, MA, USA).

To obtain Raman spectra, the samples were analyzed using a Raman spectrometer (RamanStation 400F, Perkin Elmer, USA) with point and shot capability using an excitation laser source at $785 \mathrm{~nm}$ (to avoid fluorescence excitation), 100 micron spot size, 4 shots, and $8 \mathrm{~s}$ exposition time. The obtained spectra were normalized, baseline corrected, and analyzed using SPECTRUM software (v10, Perkin Elmer, Waltham, MA, USA).

\subsection{Scanning Electron Microscopy (SEM)}

The SEM observations were carried out according to $\mathrm{Xu}$ et al. [38] with slight modification. The bacteria (MRSA ${ }^{\text {MupS }}$ and MRSA MupRL) were incubated for $18 \mathrm{~h}$ at $37^{\circ} \mathrm{C}$ on Columbia agar with $5 \%$ sheep blood. Then, the bacterial colonies were collected directly from the media surface and washed three times with $0.1 \mathrm{M}$ PBS and centrifuged (1500 rpm for $10 \mathrm{~min})$. Glutaraldehyde $(2.5 \%, v / v)$ (Chempur, Piekary Slaskie, Poland) was used to fix the bacterial cells for $4 \mathrm{~h}$ at $4{ }^{\circ} \mathrm{C}$. The samples were firstly dehydrated in a gradient ranging ethanol $(30 \%-100 \%, v / v)$ (Chempur, Poland) and then the ethanol was gradually replaced with the same ranging tert-butanol (Chempur, Poland) at room temperature. The samples were dried with vacuum freeze-drying equipment for $8 \mathrm{~h}$. Finally, bacterial cells were coated with gold for $90 \mathrm{~s}$ and a scanning electron microscope Vega 3 LMU (Tescan, Brno, Czech Republic) was used to observe.

\subsection{Statistical Analysis}

All data were expressed as mean \pm standard deviation (SD).

\section{Conclusions}

The EOCs analyzed in this study increased the mupirocin susceptibility of both mupirocin-susceptible and induced low-level mupirocin-resistant MRSA strains. This refers mostly to 1,8-cineole. Also, both staphylococcal strains revealed differences in spectroscopic profiles. These point to a cellular wall structure, however, these do not significantly affect either physical properties or mupirocin and EOC susceptibility. More precise high throughput technologies are needed to target the checkpoints crucial for staphylococcal mupirocin resistance and its control.

Author Contributions: P.K.: conceptualization, formal analysis, investigation, methodology, supervision, visualization, writing - original draft; A.P. and B.W.: formal analysis, writing—original draft; A.W.-B. and M.S.: methodology, writing—original draft; M.M.: methodology; Ł.Ł.: formal analysis, methodology, writing—original draft; B.D.: formal analysis, funding acquisition.

Acknowledgments: This research did not receive any specific grant from funding agencies in the public, commercial, or not-for-profit sectors.

Conflicts of Interest: The authors declare no conflict of interest.

\section{References}

1. Frank, D.N.; Feazel, L.M.; Bessesen, M.T.; Price, C.S.; Janoff, E.N.; Pace, N.R. The human nasal microbiota and Staphylococcus aureus carriage. PLoS ONE 2010, 5, e10598. [CrossRef] [PubMed]

2. Holtfreter, S.; Grumann, D.; Schmudde, M.; Nguyen, H.T.; Eichler, P.; Strommenger, B.; Kopron, K.; Kolata, J.; Giedrys-Kalemba, S.; Steinmetz, I.; et al. Clonal distribution of superantigen genes in clinical Staphylococcus aureus isolates. J. Clin. Microbiol. 2007, 45, 2669-2680. [CrossRef] [PubMed] 
3. El-Sayed, A.K.; Hothersall, J.; Cooper, S.M.; Stephens, E.; Simpson, T.J.; Thomas, C.M. Characterization of the mupirocin biosynthesis gene cluster from Pseudomonas fluorescens NCIMB 10586. Chem. Biol. 2003, 10, 419-430. [CrossRef]

4. World Health Organization. Global Guidelines for the Prevention of Surgical Site Infection. World Health Organization, 2016. Available online: http://www.who.int/iris/handle/10665/250680 (accessed on 10 July 2019).

5. Eltringham, I. Mupirocin resistance and methicillin-resistant Staphylococcus aureus (MRSA). J. Hosp. Infect. 1997, 35, 1-8. [CrossRef]

6. Gilbart, J.; Perry, C.R.; Slocombe, B. High-level mupirocin resistance in Staphylococcus aureus: Evidence for two distinct isoleucyl-tRNA synthetases. Antimicrob. Agents Chemother. 1993, 37, 32-38. [CrossRef]

7. Solberg, C.O. A study of carriers of Staphylococcus aureus with special regard to quantitative bacterial estimations. Acta Med. Scand. Suppl. 1965, 436, 1-96. [PubMed]

8. Reagan, D.R.; Doebbeling, B.N.; Pfaller, M.A.; Sheetz, C.T.; Houston, A.K.; Hollis, R.J.; Wenzel, R.P. Elimination of coincident Staphylococcus aureus nasal and hand carriage with intranasal application of mupirocin calcium ointment. Ann. Intern. Med. 1991, 114, 101-106. [CrossRef]

9. Sarkic, A.; Stappen, I. Essential oils and their single compounds in cosmetics-A critical review. Cosmetics 2018, 5, 11. [CrossRef]

10. Dhifi, W.; Bellili, S.; Jazi, S.; Bahloul, N.; Mnif, W. Essential oils' chemical characterization and investigation of some biological activities: A critical review. Medicines 2016, 3, 25. [CrossRef]

11. Nazzaro, F.; Fratianni, F.; Coppola, R.; De Feo, V. Essential oils and antifungal activity. Pharmaceuticals 2017, 10, 86. [CrossRef]

12. Edris, A.E. Pharmaceutical and therapeutic potentials of essential oils and their individual volatile constituents: A review. Phytother. Res. 2007, 21, 308-323. [CrossRef]

13. European Pharmacopoeia, 8th ed.; Council of Europe: Strasbourg, France, 2014.

14. Orchard, A.; van Vuuren, S. Commercial essential oils as potential antimicrobials to treat skin diseases. Evid. Based Complement. Alternat. Med. 2017, 2017, 4517971. [CrossRef]

15. Milenković, M.T.; Božić, D.D.; Slavkovska, V.N.; Lakušić, B.S. Synergistic effects of Salvia officinalis L. essential oils and antibiotics against methicillin-resistant Staphylococcus aureus. Arch. Biol. Sci. 2015, 67, 949-956. [CrossRef]

16. Mulyaningsih, S.; Sporer, F.; Zimmermann, S.; Reichling, J.; Wink, M. Synergistic properties of the terpenoids aromadendrene and 1,8-cineole from the essential oil of Eucalyptus globulus against antibiotic-susceptible and antibiotic-resistant pathogens. Phytomedicine 2010, 17, 1061-1066. [CrossRef]

17. Rudresh, M.S.; Ravi, G.S.; Motagi, A.; Alex, A.M.; Sandhya, P.; Navaneeth, B.V. Prevalence of mupirocin resistance among staphylococci, its clinical significance and relationship to clinical use. J. Lab. Physicians 2015, 7, 103-107. [CrossRef]

18. Khoshnood, S.; Heidary, M.; Asadi, A.; Soleimani, S.; Motahar, M.; Savari, M.; Saki, M.; Abdi, M. A review on mechanism of action, resistance, synergism, and clinical implications of mupirocin against Staphylococcus aureus. Biomed. Pharmacother. 2019, 109, 1809-1818. [CrossRef]

19. Grunert, T.; Jovanovic, D.; Sirisarn, W.; Johler, S.; Weidenmaier, C.; Ehling-Schulz, M.; Xia, G. Analysis of Staphylococcus aureus wall teichoic acid glycoepitopes by Fourier Transform Infrared Spectroscopy provides novel insights into the staphylococcal glycocode. Sci. Rep. 2018, 8, 1889. [CrossRef]

20. Amiali, N.M.; Golding, G.R.; Sedman, J.; Simor, A.E.; Ismail, A.A. Rapid identification of community-associated methicillin-resistant Staphylococcus aureus by Fourier transform infrared spectroscopy. Diagn. Microbiol. Infect. Dis. 2011, 70, 157-166. [CrossRef]

21. Tareb, R.; Bernardeau, M.; Amiel, C.; Vernoux, J.P. Usefulness of FTIR spectroscopy to distinguish rough and smooth variants of Lactobacillus farciminis CNCM-I-3699. FEMS Microbiol. Lett. 2017, 364. [CrossRef]

22. Kürekçi, C. Applicability of Raman spectroscopy for characterization of three major foodborne pathogens. Harran. Univ. Vet. Fak. Derg. 2016, 5, 141-145.

23. Boschetto, F.; Adachi, T.; Horiguchi, S.; Fainozzi, D.; Parmigiani, F.; Marin, E.; Zhu, W.; McEntire, B.; Yamamoto, T.; Kanamura, N.; et al. Monitoring metabolic reactions in Staphylococcus epidermidis exposed to silicon nitride using in situ time-lapse Raman spectroscopy. J. Biomed. Opt. 2018, 23, 1-10. [CrossRef] 
24. Hrubanova, K.; Krzyzanek, V.; Nebesarova, J.; Ruzicka, F.; Pilat, Z.; Samek, O. Monitoring Candida parapsilosis and Staphylococcus epidermidis biofilms by a combination of scanning electron microscopy and Raman spectroscopy. Sensors 2018, 18, 4089. [CrossRef]

25. Capobianco, J.O.; Doran, C.C.; Goldman, R.C. Mechanism of mupirocin transport into sensitive and resistant bacteria. Antimicrob. Agents Chemother. 1989, 33, 156-163. [CrossRef]

26. Hill, R.L. The bioavailability of mupirocin in nasal secretions in vitro. J. Clin. Pathol. 2002, 55, $233-235$. [CrossRef]

27. Kwiatkowski, P.; Grygorcewicz, B.; Pruss, A.; Wojciuk, B.; Dołęowska, B.; Giedrys-Kalemba, S.; Sienkiewicz, M.; Wojciechowska-Koszko, I. The effect of subinhibitory concentrations of trans-anethole on antibacterial and antibiofilm activity of mupirocin against mupirocin-resistant Staphylococcus aureus strains. Microb. Drug Resist. 2019. [CrossRef]

28. Hendry, E.R.; Worthington, T.; Conway, B.R.; Lambert, P.A. Antimicrobial efficacy of eucalyptus oil and 1,8-cineole alone and in combination with chlorhexidine digluconte against microorganisms grown in planktonic and biofilm cultures. J. Antimicrob. Chemother. 2009, 64, 1219-1225. [CrossRef]

29. Kifer, D.; Mužinić, V.; Klarić, M.Š. Antimicrobial potency of single and combined mupirocin and monoterpenes, thymol, menthol and 1,8-cineole against Staphylococcus aureus planktonic and biofilm growth. J. Antibiot. (Tokyo) 2016, 69, 689-696. [CrossRef]

30. Şimşek, M.; Duman, R. Investigation of effect of 1,8-cineole on antimicrobial activity of chlorhexidine gluconate. Pharmacognosy Res. 2017, 9, 234-237. [CrossRef]

31. Xu, J.; Hu, Z.Q.; Wang, C.; Yin, Z.Q.; Wei, Q.; Zhou, L.J.; Li, L.; Du, Y.H.; Jia, R.Y.; Li, M.; et al. Acute and subacute toxicity study of 1,8-cineole in mice. Int. J. Clin. Exp. Pathol. 2014, 7, 1495-1501.

32. Yadav, M.K.; Chae, S.W.; Im, G.J.; Chung, J.W.; Song, J.J. Eugenol: A phyto-compound effective against methicillin-resistant and methicillin-sensitive Staphylococcus aureus clinical strain biofilms. PLoS ONE 2015, 10, e0119564. [CrossRef]

33. Trombetta, D.; Castelli, F.; Sarpietro, M.G.; Venuti, V.; Cristani, M.; Daniele, C.; Saija, A.; Mazzanti, G.; Bisignano, G. Mechanisms of antibacterial action of three monoterpenes. Antimicrob. Agents Chemother. 2005, 49, 2474-2478. [CrossRef]

34. Methods for Dilution Antimicrobial Susceptibility Test for Bacteria that Grow Aerobically, Approved Standard; M07-A9; Clinical and Laboratory Standard Institute (CLSI): Wayne, PA, USA, 2012.

35. Kwiatkowski, P.; Pruss, A.; Grygorcewicz, B.; Wojciuk, B.; Dołegowska, B.; Giedrys-Kalemba, S.; Kochan, E.; Sienkiewicz, M. Preliminary study on the antibacterial activity of essential oils alone and in combination with gentamicin against extended-spectrum $\beta$-lactamase-producing and New Delhi metallo- $\beta$-lactamase-1-producing Klebsiella pneumoniae isolates. Microb. Drug Resist. 2018, 24, 1368-1375. [CrossRef]

36. Lee, A.S.; Gizard, Y.; Empel, J.; Bonetti, E.J.; Harbarth, S.; François, P. Mupirocin-induced mutations in ileS in various genetic backgrounds of methicillin-resistant Staphylococcus aureus. J. Clin. Microbiol. 2014, 52, 3749-3754. [CrossRef]

37. Yap, P.S.; Lim, S.H.; Hu, C.P.; Yiap, B.C. Combination of essential oils and antibiotics reduce antibiotic resistance in plasmid-conferred multidrug resistant bacteria. Phytomedicine 2013, 20, 710-713. [CrossRef]

38. Xu, C.; Li, J.; Yang, L.; Shi, F.; Yang, L.; Ye, M. Antibacterial activity and a membrane damage mechanism of Lachnum YM30 melanin against Vibrio parahaemolyticus and Staphylococcus aureus. Food Control 2017, 73, 1445-1451. [CrossRef]

Sample Availability: Samples of the compounds (1,8-cineole, eugenol, carvacrol, linalool, (-)-menthone, linalyl acetate and trans-anethole) are available from the authors.

(C) 2019 by the authors. Licensee MDPI, Basel, Switzerland. This article is an open access article distributed under the terms and conditions of the Creative Commons Attribution (CC BY) license (http://creativecommons.org/licenses/by/4.0/). 